\title{
Numerical Study on Flow Focusing in Axisymmetric Microchannel
}

\author{
X. P. Chen ${ }^{1, *}$ \\ ${ }^{1}$ School of Mech., Civil Engg and Architecture, Northwestern Polytech. University, Xi'an 710072, \\ China \\ Email: xchen76@nwpu.edu.cn
}

\begin{abstract}
Flow focusing normally refers to two immiscible liquids (phase 1 and 2) are extracted into a small orifice while the liquid interface pinches off due to capillary instability or forces balance among driven pressure, interfacial tension etc [1]. The idea stimulated a mass of studies of flow focusing in microchannels [2], which show excellent controllability on the droplet sizes and drop manipulation.

In this study, we present numerical simulation on the microscale flow-focusing under axisymmetric conditions with commercial software, CFDRC-ACE (VOF module). Two interfacial breakup modes, dripping mode and jetting mode, are observed (as shown in the Figures 1,2 below). According to our results, the transition from dripping mode to jetting mode is motivated by increasing either inner or outer flow rate. In another words, increasing the kinetic energy contributes to this transition. The critical transition Weber's number $\left(W e=\rho V^{2} a / \sigma\right.$, where $a$ is the characteristic length, $\rho$ the density, $V$ the velocity, $\sigma$ the interfacial tension) is found to be around 1. For dripping mode, the droplet size analyses indicate that the viscous drag dominates the breakup, while, for jetting mode, the jet length implies that capillary instability dominates the breakup. The work in the future, both theoretical and technical, is prospected in this paper as well.
\end{abstract}

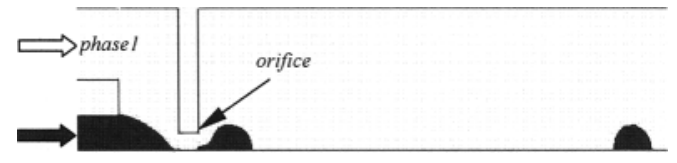

Figure 1: Dripping Mode. The diameter of the nozzle and inner inlet channel are $50 \mu \mathrm{m}$ and $100 \mu \mathrm{m}$ respectively. The outer and inner liquids are water and silicon oil, respectively. Outer and inner inflow velocity are 5 and $7.5 \mathrm{~mm} / \mathrm{sec}$. respectively

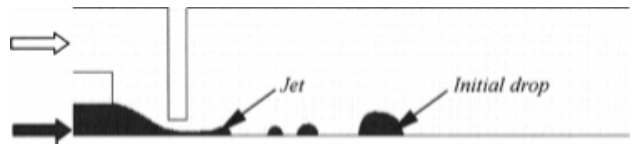

Figure 2: Jetting Mode. The outer and inner inflow velocity are $18 \mathrm{~mm} / \mathrm{sec}$ and $8 \mathrm{~mm} / \mathrm{sec}$ respectively

\section{REFERENCES}

1. Gannan-Calvo AM, Gordillo JM. Perfectly monodisperse microbubbling by capillary flow focusing. Phys. Rev. Lett., 2001; 87: 274501

2. Anna SL, Bontoux N, Stone HA. Formation dispersions using "flow focusing" in microchannels. Appl. Phys. Lett., 2003; 82: 364-366 